\title{
Temperature Dependence of Exchange Anisotropy of FeNi/FeMn Bilayers
}

\author{
R. Żuberek, M. Gutowski, H. Szymczak \\ Institute of Physics of the Polish Academy of Sciences \\ al. Lotników 32/46, 02-668 Warsaw, Poland
}

\section{S.L. Gnatchenko, D.N. Merenkov and A.N. Bludov}

B. Verkin Institute for Low Temperature Physics and Engineering

National Academy of Sciences of Ukraine

Lenin Ave. 47, 611093 Kharkov, Ukraine

\begin{abstract}
The influence of the bilinear and biquadratic coupling on the exchange anisotropy of the ferromagnetic/antiferromagnetic bilayer was studied by variable angle and temperature dependent ferromagnetic resonance. From the angular dependence of the resonance field the bilinear and biquadratic exchange coupling constants were determined.
\end{abstract}

PACS numbers: 73.21.Ac, 75.30.Et, 75.70.-i, 76.50.+g

\section{Introduction}

In thin films composed of a ferromagnetic (FM) and an antiferromagnetic (AFM) layers, a unidirectional anisotropy occurs when spins at the interface of FM and AFM layers are exchange coupled. The unidirectional anisotropy is responsible for a distinctive shift of a hysteresis loop away from zero along the exciting field axis. The basic physics of FM/AFM exchange coupling remains poorly understood $[1,2]$. In this work, in order to clarify the nature of FM/AFM exchange coupling we have performed temperature dependent ferromagnetic resonance (FMR) measurements on $\mathrm{FeNi} / \mathrm{FeMn}$ bilayers taking into account the bilinear and biquadratic exchange coupling terms.

\section{Experimental}

The $\mathrm{FeNi}(50 \AA) / \mathrm{FeMn}(50 \AA)$ bilayer film was produced by $\mathrm{RF}$ ion sputtering in the Ar atmosphere [3]. An Si plate cut in the (100) plane was used as a substrate kept at room temperature. The $\mathrm{Fe}_{50} \mathrm{Mn}_{50} \mathrm{AFM}$ was deposited on the permalloy 
$\mathrm{Fe}_{20} \mathrm{Ni}_{80}$ layer, both in the field $H_{\mathrm{f}} \approx 30$ Oe directed in the substrate plane. The $\mathrm{FeNi} / \mathrm{FeMn}$ film had protecting surface and buffer gold layers $30 \AA$ thick. The deposition of the two-layer film in the magnetic field induced uniaxial anisotropy (its easy axis parallel to $\mathrm{H}_{\mathrm{f}}$ ) and unidirectional exchange anisotropy in the FM layer.

The FMR measurements were performed at X band $(9.248 \mathrm{GHz})$ and in the external field in the plane made by FM layer easy axis and the direction normal to the film. The sample was cooled in a field of 100 Oe aligned with the easy axis of the FM layer prior to the measurements. The resulting exchange bias effect, as seen in the FMR experiment, together with its rapid disappearance when the temperature rises, is shown in Fig. 1.
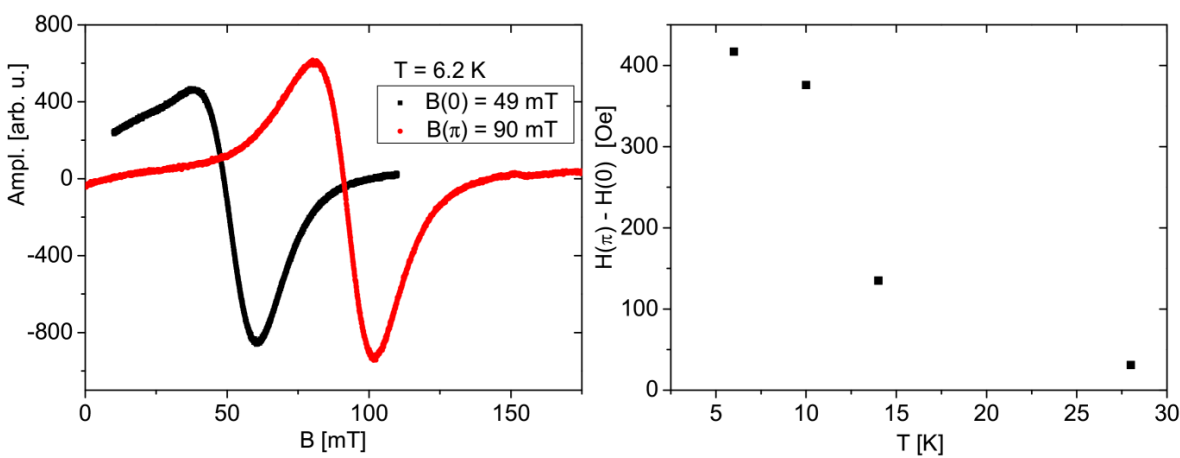

Fig. 1. Left: resonance lines observed for external field in sample plane - along the easy axis and in the opposite direction; right: the exchange field, $\mid H_{\mathrm{res}}(\varphi=\pi)-H_{\mathrm{res}}(\varphi=$ $0)\left.\right|_{\theta=\pi / 2}$ rapidly decreases with increasing temperature.

\section{FMR conditions}

We use the model for FM/AFM bilayers introduced by Hu et al. [4]. In spherical coordinates, the total energy per unit area of the FM/AFM bilayers can be written as

$$
\begin{aligned}
E= & \left\{2 \pi M_{\mathrm{FM}}^{2} \cos ^{2} \theta-M_{\mathrm{FM}} H\left[\sin \theta \sin \theta_{\mathrm{H}} \cos \left(\varphi-\varphi_{\mathrm{H}}\right)+\cos \theta \cos \theta_{\mathrm{H}}\right]\right. \\
& \left.-K_{\mathrm{FM}}^{\mathrm{out}} \cos ^{2} \theta-K_{\mathrm{FM}}^{\mathrm{in}} \sin ^{2} \theta \cos ^{2} \varphi\right\} t_{\mathrm{FM}}-\sigma_{\mathrm{AFM}} \sin \theta_{\mathrm{A}} \cos \varphi_{\mathrm{A}} \\
& -J_{1}\left[\sin \theta \sin \theta_{\mathrm{A}} \cos \left(\varphi-\varphi_{\mathrm{A}}\right)+\cos \theta \cos \theta_{\mathrm{A}}\right] \\
& +J_{2}\left[\sin \theta \sin \theta_{\mathrm{A}} \cos \left(\varphi-\varphi_{\mathrm{A}}\right)+\cos \theta \cos \theta_{\mathrm{A}}\right]^{2},
\end{aligned}
$$

where the interaction of the external field with the AFM layer is neglected.

The $x$ axis is the easy direction of the FM layer and the $z$ axis is perpendicular to the film. The angle pairs: $(\theta, \varphi),\left(\theta_{\mathrm{H}}, \varphi_{\mathrm{H}}\right)$ and $\left(\theta_{\mathrm{A}}, \varphi_{\mathrm{A}}\right)$ describe the orientation of the FM moment, $M_{\mathrm{FM}}$, external field, $H$, and the net AFM sublattice moment at the interface, respectively. $K_{\mathrm{FM}}^{\text {out }}\left(K_{\mathrm{FM}}^{\mathrm{in}}\right)$ is the out-of-plane 
(in-plane) FM anisotropy constant, $\sigma_{\mathrm{AFM}}$ - the domain wall energy in the AFM layer, $J_{1}$ and $J_{2}$ are the bilinear and biquadratic interfacial coupling constants, respectively.

The ferromagnetic resonance condition reads

$$
t_{\mathrm{FM}}^{2}\left(\frac{\omega}{\gamma}\right)^{2}=\frac{1}{M_{\mathrm{FM}}^{2} \sin ^{2} \theta}\left(\frac{\partial^{2} E}{\partial \theta^{2}} \frac{\partial^{2} E}{\partial \varphi^{2}}-\frac{\partial^{2} E}{\partial \theta \partial \varphi}\right)
$$

at the equilibrium position(s) of the entire system, to be found by solving the equation $\nabla E\left(\theta, \varphi, \theta_{\mathrm{A}}, \varphi_{\mathrm{A}}\right)=(0,0,0,0)$.

\section{Details of data analysis and results}

The spectra were simulated using highly accurate interval techniques. The essence of interval calculus is to evaluate the ranges of arithmetic expressions given the ranges of their individual constituents. The advantages of this approach are: the problem is attacked in its original, undistorted form, with no simplifications or approximations, and no resonance is ever missed. All the possible numerical inaccuracies and roundings are always correctly accounted for.

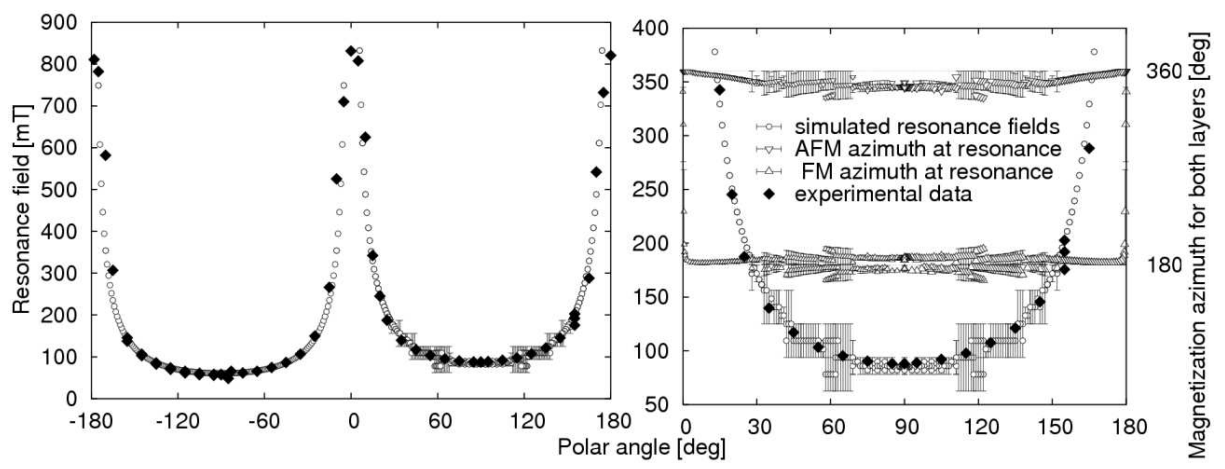

Fig. 2. Simulated (open circles) and measured (diamonds) resonance fields vs. external field orientation. Left part of left figure corresponds to the azimuthal angle $\varphi=0$, and right - to $\varphi=\pi$. More details on the right: at some orientations there are 2, or sometimes even 4 , distinct equilibrium positions of $M_{\mathrm{FM}}$ and $M_{\mathrm{AFM}}$ vectors, resulting in 1 or 2 different resonance fields.

Simulated spectra, (Fig. 2), obtained for various sets of unknown parameters, were later analyzed in a classical way, i.e. using $\chi^{2}$ methodology. The following numerical values of the interesting physical parameters were found at $T=6 \mathrm{~K}$ : $g_{\text {eff }}=\gamma / \mu_{\mathrm{B}}=2.026 \pm 0.005, \sigma_{\mathrm{AFM}}=(0.22 \pm 0.04) \mathrm{erg} / \mathrm{cm}^{2}, J_{1}=(0.089 \pm$ $0.002) \mathrm{erg} / \mathrm{cm}^{2}$, and $J_{2}=(0.037 \pm 0.001) \mathrm{erg} / \mathrm{cm}^{2}$. It is impossible to find the value of $K_{\mathrm{FM}}^{\mathrm{in}}$ from our measurements and therefore we had to fix it at $270 \mathrm{erg} / \mathrm{cm}^{3}$ during calculations. Similarly, due to the identical angular dependence, we cannot 
separate $M_{\mathrm{FM}}$ from $K_{\mathrm{FM}}^{\text {out }}$. Their mixture, namely $\sqrt{M_{\mathrm{FM}}^{2}-K_{\mathrm{FM}}^{\text {out }} / 2 \pi} \approx M_{\mathrm{FM}}$, was found to be equal to $(1294.0 \pm 4.6)$ Oe.

According to Hu's model [4], the exchange anisotropy is defined as $J_{\mathrm{FMR}}=$ $\frac{1}{2}\left(M_{\mathrm{FM}} t_{\mathrm{FM}}\right)\left[H_{\text {res }}(\pi)-H_{\text {res }}(0)\right]$, but may be also expressed as

$$
J_{\mathrm{FMR}}=\left\{\begin{array}{lll}
\frac{J_{1} \sigma_{\mathrm{AFM}}^{2}}{\left(2 J_{2}+\sigma_{\mathrm{AFM}}\right)^{2}-J_{1}^{2}} & \text { if } & J_{1}<\sigma_{\mathrm{AFM}}+2 J_{2} \\
\frac{\left(J_{1}-2 J_{2}\right)^{2} \sigma_{\mathrm{AFM}}}{\left(J_{1}-2 J_{2}\right)^{2}-\sigma_{\mathrm{AFM}}^{2}} & \text { if } & J_{1}>\sigma_{\mathrm{AFM}}+2 J_{2} .
\end{array}\right.
$$

In our case the first expression evaluates to $J_{\mathrm{FMR}}=0.063 \mathrm{erg} / \mathrm{cm}^{2}$, while the calculations involving the fitted parameters yield $J_{\mathrm{FMR}}=0.055 \mathrm{erg} / \mathrm{cm}^{2}$. As a side effect, our computations revealed that FMR occurs at local rather than global free energy minimum. For majority of cases, aside from those when the field is nearly perpendicular to the sample plane, the energy at resonance locates itself almost precisely halfway between global minimum and global maximum.

\section{Conclusions}

Our experiment, and its analysis, show that the free energy landscape is qualitatively different when the external field is directed along the earlier applied exchange-biasing field compared to the situation when those directions are mutually opposite. Namely, the landscape is quite smooth when both FM and AFM magnetizations are parallel and there is a kind of magnetic friction between the two layers, caused by the presence of many local energy minima, when they are antiparallel.

\section{References}

[1] J. Nogues, I.K. Schuller, J. Magn. Magn. Mater. 192, 203 (1999).

[2] A.E. Berkowitz, K. Takano, J. Magn. Magn. Mater. 200, 552 (1999).

[3] S.L. Gnatchenko, D.N. Merenkov, A.N. Bludov, V.V. Pishko, Yu.A. Shakhayeva, M. Baran, R. Szymczak, V.A. Novosad, J. Magn. Magn. Mater. 307, 263 (2006).

[4] Jing-gou Hu, Guojun Jin, R.L. Stamps, Yu-qiang Ma, J. Magn. Magn. Mater. 301, 238 (2006). 